\title{
La storia della filosofia secondo Reinhold all'inizio del XIX secolo
}

\author{
The history of philosophy at the beginning \\ of 19th century according to Reinhold. \\ A história da filosofia segundo Reinhold \\ no início do século XIX
}

\section{Pierluigi Valenza*}

Università degli Studi “Sapienza" di Roma, Roma, Italia

\section{Riassunto}

Beyträge zur leichtern Uebersicht des Zustandes der Philosophie beym Anfange des 19. Jahrhunderts, i cui sei tomi furono publicati da K.L. Reinhold tra il 1801 e il 1803 offrono in alcuni dei loro saggi um panorama della filosofia all'inizio del secolo XIX. II punto di vista di Reinhold è influenzato dalla sua conversione al realismo logico e, di conseguenza, è orientato criticamente contro la filosofia trascendentale kantiana, così come contro l'idealismo fichtiano e schellinghiano. Nella prima parte di questo articolo presenterò l'idea reinholdiana di storia della filosofia come processo orientato verso una rivoluzione definitiva e ultima nella filosofia. Sosterrò che l'idea reinholdiana di una rivoluzione nella

*PV: Dottore in Filosofia, e-mail: pierluigi.valenza@uniroma1.it 
filosofia appare in un orizzonte articolato in cui sono coinvolte scoperta e fondazione della conoscenza. Nella seconda parte del saggio chiarirò i criteri reinholdiani per valutare la storia della filosofia e, in particolare, la differenza tra "vero" e "vero originario", atrraverso la quale Reinhold interpreta Platone, Leibniz e il realismo logico come punti fondamentali della filosofia, ma anche attraverso un riferimento particolare a Descartes. La terza e ultima parte di questo articolo è volta a presentare la critica di Reinhold alla filosofia kantiana, fichtiana e schellinghiana. Per Reinhold la soggettività consiste in una attività libera dell'io che astrae da oggetti e riflette su se stessa e, infine, cade in una illusione psicologica. Allo stesso modo l'identà schellinghiana di soggettività e oggettività potrebbe apparire molto vicina al realismo logico. L'idea reinholdiana della filosofia contemporanea è tuttavia orientata a un confronto critico e rigoroso con la filosofia tarda di Schelling, unita, per altro verso, a una discussione della opposizione tra conoscenza e fede.

Parole chiave: Reinhold. Realismo. Storia della filosofia. Kant. Idealismo.

\section{Abstract}

The journal Beyträge zur leichtern Uebersicht des Zustandes der Philosophie beym Anfange des 19. Jahrhunderts, whose six volumes were edited by K. L. Reinhold from 1801 until 1803, gives in some of its essays an outlook of the philosophy at the beginning of 19th century. Reinhold's point of view is driven by his conversion to logical realism and consequently critically turned against Kantian transcendental philosophy as well as Fichtian and Schellinghian Idealism. In the first part of the essay Reinhold's idea of history of philosophy as process toward a last and definitive revolution in philosophy is expounded. I argue that Reinhold's idea of a revolution in philosophy looks at an articulated step in which discovery and foundation of knowledge are involved. Anyway, the history of philosophy according to Reinhold is possible from a philosophical point of view, that one of the final revolution in philosophy. In the second part of the essay Reinhold's criterions to evaluate history of philosophy are explained, particularly the difference between "true" and "originally true" through which Reinhold considers Plato, Leibniz and logical realism as peaks of history of philosophy, but with a special regard also to Descartes. The third and last part of the essay deals with Reinhold's criticism toward Kantian philosophy and Idealism. Reinhold conceives subjectivity as the common principle of Kantian, Fichtian and Schellinghian philosophy. Subjectivity according to Reinhold consists in a free activity of the Self abstracting from the objects and reflecting on itself and thus finally in a psychological deception. Nevertheless, Schellinghian 
identity of subjectivity and objectivity could seem close to logical realism. Reinhold's idea of contemporary philosophy looks at a closed confrontation with Schelling's last philosophy on one hand and a debate with Jacobi's theory of faith opposed to knowledge on the other.

Keywords: Reinhold. Realism. History of philosophy. Kant. Idealism.

\section{Resumo}

A revista Beyträge zur leichtern Uebersicht des Zustandes der Philosophie beym Anfange des 19. Jahrhunderts, cujos seis tomos foram publicados por K. L. Reinhold entre 1801 e 1803, oferece em alguns ensaios um panorama da filosofia no começo do século XIX. O ponto de vista de Reinhold é influenciado pela conversão dele ao realismo lógico e, por conseguinte, é virado criticamente contra a filosofia transcendental kantiana, assim como contra o idealismo fichtiano e scellinguiano. Na primeira parte deste artigo irei apresentar a ideia reinholdiana de história da filosofia como processo orientado para uma revolução definitiva e última na filosofia. Irei sustentar que a ideia reinholdiana de uma revolução na filosofia aparece como nível articulado em que estão envolvidas descoberta e fundação do conhecimento. Para Reinhold a história da filosofia é possivel de um ponto de vista filosófico, qual seja o da revolução final na filosofia. Na segunda parte do ensaio irei esclarecer os critérios reinholdianos para avaliar a história da filosofia, e em especial a diferença entre "verdadeiro" e "originariamente verdadeiro" através da qual Reinhold considera Platão, Leibniz e o realismo lógico como pontos fundamentais da filosofia, mas com uma referência especial para Descartes também. A terceira e última parte deste artigo visa apresentar a crítica de Reinhold à filosofia kantiana e ao idealismo. Reinhold conceitua a subjetividade como princípio comum da filosofia kantiana, fichtiana e schellinguiana. Para Reinhold a subjetividade consiste em uma atividade livre do si mesmo que abstrai dos objetos e reflete em si mesma e, por fim, cai em uma ilusão psicológica. Igualmente a identidade schellinguiana de subjetividade e objetividade pode aparecer próxima do realismo lógico. A ideia da filosofia contemporânea de Reinhold visa uma confrontação rigorosa com a filosofia tardia de Schelling e, por outro lado, uma discussão da teoria reinholdiana da fé oposta ao conhecimento.

Palavras-chave: Reinhold. Realismo. História da filosofia. Kant. Idealismo. 


\section{Introduzione}

L'inizio di un nuovo secolo costituisce sempre un momento temporale che suggerisce bilanci e delineazione di prospettive. La rivista cui Karl Leonhard Reinhold dà vita e che cura componendo la maggior parte dei saggi, i Beyträge zur leichtern Uebersicht des Zustandes der Philosophie beym Anfange des 19. Jahrhunderts, uscita in sei quaderni tra 1801 e 1803, già nel titolo appare rispondere a quest'istanza ovvia e tuttavia non così diffusa come si potrebbe pensare. Meno profilata in questa direzione, anche se non priva di sguardo storico, è la rivista coeva cui danno vita Schelling e Hegel, il Kritisches Journal, più orientato a definire una critica filosofica, ma anche poi a presentare gli sviluppi della filosofia idealistica dei due autori, allora in stretta vicinanza filosofica oltre che biografica. Entrambe le riviste erano accomunate dall'esigenza di definire lo stato della filosofia idealistica all'epoca. Tuttavia, e su questo si concentrerà l'attenzione di questo saggio, i Beyträge editi da Reinhold, e soprattutto alcuni dei contributi di Reinhold stesso, si caratterizzano per una spiccata sensibilità storico-filosofica. Il genere rimaneva recente se non recentissimo, pensando al fiorire di storie della filosofia dell'ultimo decennio del Settecento, legate alla rivoluzione filosofica introdotta da Kant nel confronto con la tradizione filosofica precedente ${ }^{1}$. Da questo lato forse il precedente più vicino ai Beyträge reinholdiani lo si può individuare nei Beiträge zur Geschichte der Philosophie, curati da Georg Gustav Fülleborn tra 1796 e 1799, e che ospitano l'intervento teorico più significativo dello stesso Reinhold sulla concezione della storia della filosofia prima dei Beyträge zur leichtern Uebersicht, il saggio Über den Begriff der Geschichte der Philosophie. Eine akademische Vorlesung ${ }^{2}$.

\footnotetext{
Emblematica la proposta di concorso da parte dell'Accademia delle scienze di Berlino nel 1791 avente come tema i progressi compiuti dalla metafisica dai tempi di Leibniz e Wolff, vero e proprio concorso di idee sullo stato della filosofia tedesca dopo la svolta kantiana. Basterà poi ricordare l'uscita nell'ultimo decennio del Settecento di opere in più volumi di storia della filosofia, quali Geist der spekulativen Philosophie, di Dietrich Tiedemann, e Geschichte der Philosophie di Wilhelm Gottlob Tennemann.

2 II saggio esce alle pagine 3-36 dei Beiträge zur Geschichte der Philosophie, a cura di G. G. Fülleborn, I u. II Stück, nel 1796. Della rivista escono in totale, fino al 1799, dodici volumi con contributi di autori vari, ma prevalentemente dello stesso Fülleborn, che spaziano da saggi e traduzioni dedicati al pensiero antico fino alla filosofia dell'epoca e a contributi sul concetto di storia della filosofia come quello reinholdiano.
} 
Reinhold, proprio nel senso teorico di questo saggio, contribuisce in modo decisivo alla perimetrazione del significato filosofico della storia della filosofia, del tutto dentro il dibattito sviluppato dai grandi autori dell'idealismo tedesco, da Schelling allo Hegel che nel suo primo testo a stampa discute ampiamente le tesi di Reinhold nei Beyträge zur leichtern Uebersicht ${ }^{3}$.

Nei quaderni della rivista, e segnatamente nei saggi più esplicitamente storico-filosofici, Reinhold si produce in una storia della filosofia sul campo, ovvero presenta i maggiori filosofi dell'età moderna fino all'età a lui contemporanea, riassumendone e commentandone le tesi principali ${ }^{4}$. Una ricostruzione storica che è però fortemente filosofica, guidata cioè da precise tesi interpretative, alcune anche del tutto nuove rispetto alle sue numerose ricostruzioni precedenti, e innovative anche rispetto a visioni consolidate sulla filosofia moderna.

Nell'arrivare alla filosofia contemporanea, Reinhold entra in una serrata discussione critica con gli esiti della filosofia tedesca, soprattutto rappresentati all'epoca dai sistemi filosofici di Fichte e Schelling. Qui opera il nuovo sguardo che ispira l'intera rivista e i contributi teoretici, storico-filosofici e polemici di Reinhold, vale a dire la conversione al realismo logico a seguito della lettura del Grundriss der ersten Logik di Christoph Gottfried Bardili e dell'adesione originale e non acritica al disegno ivi contenuto di una logica-ontologia avversa al trascendentalismo kantiano e fichtiano ${ }^{5}$. Questo punto di vista ispiratore dell'intera impresa dovrà necessariamente costituire qui il punto di partenza perché è la lente operante ai diversi livelli ora elencati: è l'approdo del

3 II riferimento è alla prima monografia hegeliana: Differenz des Fichte'schen und Schelling'schen Systems der Philosophie, in Beziehung auf Reinhold's Beyträge zur leichtern Uebersicht des Zustandes der Philosophie zu Anfang des neunzehnten Jahrhunderts, 1stes Heft, pubblicata presso l'editore Seidler di Jena nel 1801.

4 I saggi tematicamente storico-filosofici sono le tre parti de Die erste Aufgabe der Philosophie, in ihren merkwürdigsten Auflösungen, seit der Wiederherstellung der Wissenschaften, in Reinhold (1801-1803, citato di qui in avanti con l'abbreviazione B seguita dal numero romano del volume), rispettivamente B I p. 1-65, B II p. 1-71 (dedicato al periodo 1781-1800) e B III p. 210-236, in cui è trattato soltanto Bouterwek.

5 Cf. BARDILI, 1800. Bardili era un pensatore allora pressoché sconosciuto, anche se autore di altri testi filosofici, e docente allo Stift di Tubinga, quindi docente di Hegel e di Schelling, di quest'ultimo anche cugino. Reinhold legge il Grundriss al momento della sua pubblicazione, avvenuta in realtà già nell'autunno del 1799, e lo studia intensamente fino alla primavera del 1800 recensendolo sull'Allgemeine Literatur-Zeitung. 
realismo logico la meta da cui leggere il senso dell'intera storia della filosofia, nelle sue rivoluzioni mancate fino alla rivoluzione ultima; è sempre il realismo nell'interpretazione originale di Reinhold a fornire le linee portanti per leggere i momenti topici della storia della filosofia, della filosofia moderna e della filosofia in generale; infine il realismo forma la base per criticare gli esiti dell'idealismo, il soggettivismo fichtiano, ma anche l'apparente superamento del soggettivismo fichtiano nella filosofia dell'identità di Schelling.

\section{La storia della filosofia come progresso fino alla rivoluzione riuscita}

Nella Premessa ai Beyträge Reinhold introducendo il punto di vista che sarà esposto nel complesso dei contributi della rivista e che guiderà anche il suo sguardo storico, dichiara al pubblico filosofico il suo errore circa l'idea di una definitiva rivoluzione in filosofia annunciata e sostenuta in tutta la sua produzione precedente: la rivoluzione in filosofia non è quella kantiana annunciata nei suoi Briefe über die Kantische Philosophie, né quella perseguita successivamente tentando una fondazione rigorosa del criticismo kantiano attraverso la teoria della rappresentazione, né, in ultimo, quella che aveva portato Reinhold ad aderire alla Wissenschaftslehre fichtiana dismettendo l'intera sua costruzione filosofica precedente ${ }^{6}$. Questo completo rovesciamento di prospettiva è riportato da Reinhold all'errore di tutti i riformatori e più in generale di tutta la filosofia, quello di aver individuato nella metafisica il campo decisivo del confronto filosofico e non piuttosto nella logica, secondo il pregiudizio per il quale - e Reinhold riprende la formulazione del Kant della Kritik der reinen Vernunft - la logica dopo Aristotele non avrebbe conosciuto progressi né sarebbe suscettibile di progressi. Questo punto di vista per Reinhold è stato definitivamente messo in questione da Bardili nel suo Grundriss der ersten Logik, nel momento in cui Bardili mette in dubbio la validità delle leggi logiche in quanto

Cf. $B /$ p. III-IV.

7 Cf. B / p. VIII che cita dalla premessa alla seconda edizione della Kritik der reinen Vernunft: cf. KANT, 1956, B VIII.

Rev. Filos., Aurora, Curitiba, v. 30, n. 51, p. 727-750, set./dez. 2018 
formali, ovvero come leggi del pensare di un soggetto pensante. Senza naturalmente poter minimamente entrare nel merito della prospettiva bardiliana, qui basterà ricordare che Bardili indica il cuore della sua opera in una dottrina della ragione come unione di logica e metafisica e questa prende le mosse da un pensiero concepito assolutamente, cioè non proprio di un soggetto pensante, e non suscettibile di negazione, immutabile nel suo perenne potersi ripetere come operazione assoluta, oggettiva, regola che fonda il singolo pensare e pensato ${ }^{8}$. A questo carattere assoluto del pensiero in Bardili fa riferimento Reinhold quando afferma, sempre nella Premessa al primo quaderno della rivista, che per il nuovo punto di vista di quest'ultima e definitiva rivoluzione filosofica «certezza logica, metafisica e matematica si risolvono in un'unica e medesima certezza reale, e verità soggettiva e oggettiva in un'unica e medesima verità reale» ${ }^{9}$.

Se quindi siamo in presenza di un completo capovolgimento di prospettiva rispetto alle rivoluzioni filosofiche preconizzate precedentemente, a prima vista rimane ferma l'idea del corso della storia della filosofia che trovi la sua conclusione in un ultimo e definitivo sistema filosofico. È questa la visione che fissa polemicamente Hegel nella sua discussione critica dell'idea di storia della filosofia di Reinhold nella Differenzschrift: la storia della filosofia come sequela di punti di vista peculiari che approssimerebbero la soluzione del compito della filosofia in virtù dell'invenzione di nuove tecniche fino a «una tecnica ultima "di valore universale", mediante la quale, per chiunque volesse familiarizzarsi con essa, l'opera si produrrebbe da sé» ${ }^{10}$. Salvo la forzatura interpretativa sull'idea di filosofia come tecnica, Hegel coglie nel segno circa l'idea della storia della filosofia come approssimazione ad un ultimo tentativo riuscito: in avvio della prima parte, nel primo quaderno dei Beyträge, del saggio Die erste Aufgabe der Philosophie, in ihren merkwürdigsten Auflösungen, seit der Wiederherstellung der Wissenschaften, Reinhold dopo aver definito come il primo e più essenziale compito della filosofia

\footnotetext{
8 Cf. per quest'impostazione in particolare i primi paragrafi del Grundriss, BARDILI, 1800, p. 3-4.

$9 B / p$. IX-X. Se non diversamente segnalato i corsivi sono da intendersi sempre di Reinhold.

10 HEGEL, 1968, p. 10.
} 
«la fondazione della realtà della conoscenza» ${ }^{11}$, mostra di intendere i diversi tentativi di risposta dei filosofi come tesi ad una soluzione ultima:

Anche se falliti, almeno i più notevoli tra questi tentativi paiono essere stati inevitabili e indispensabili nel provocare, come esercizi preparatori, quello che poi in futuro riuscirà, se per l'umanità non è destinato altrimenti. Se il progresso del filosofare impegnato nella ricerca della filosofia come scienza e lo scopo di questo progresso non sono una pura pretesa, allora nel filo conduttore di quei diversi tentativi debbono preannunciarsi in modo evidente questo progresso e l'approssimazione allo scopo ${ }^{12}$.

È interessante notare che nel momento stesso in cui Reinhold legge la storia della filosofia come approssimazione allo scopo, con una punta di scetticismo lascia aperta l'ipotesi che l'ultimo e riuscito tentativo possa anche non darsi. Dietro quest'ipotesi c'è qualcosa di più profondo che la consapevolezza di assumere un punto di vista minoritario a fronte degli sviluppi della filosofia idealistica contemporanea, un'idea che contribuisce a correggere la concezione di "tecnica" attribuitagli da Hegel. Mi riferisco alla convinzione che le rivoluzioni in filosofia difficilmente si concentrino in un unico sistema, nell'edificio di pensiero di un solo filosofo, ma in un'articolazione che dal punto di vista rivoluzionario prosegue in direzione della sua illustrazione e fondazione. Anche per le rivoluzioni precedenti ripercorse da Reinhold con lui stesso come co-protagonista non era andata altrimenti: in modo diverso i Briefe über die Kantische Philosophie e il Versuch einer neuen Theorie des menschlichen Vorstellungsvermögens avevano avuto la funzione di spiegare e diffondere prima, di fondare rigorosamente poi, la rivoluzione kantiana. E nella seconda versione, pubblicata nel secondo volume dell'Auswahl vermischter Schriften, della risposta al quesito bandito dall'Accademia di Berlino sui progressi della metafisica dall'epoca di Leibniz e Wolff, Reinhold aveva dovuto sviluppare una vera e propria rassegna dei diversi indirizzi di pensiero suscitati dalla

11 B/p. 2.

12 B/p. 4-5. 
rivoluzione kantiana, chiaro segno di come questa non avesse affatto costituito la fine dei tentativi di soluzione del compito della filosofia ${ }^{13}$.

Se rispetto alla rivoluzione kantiana Reinhold aveva organizzato in modo più strettamente teorico e logico i diversi indirizzi filosofici, raggruppandoli in grandi classi come scetticismo, soprannaturalismo, naturalismo, che tenevano insieme pensatori di epoche diverse, rispetto alla rivoluzione costituita dal realismo logico apre maggiormente lo sguardo alla distensione temporale che segna le differenze fra filosofia antica e moderna e fra le varie fasi della filosofia moderna. Forse anche in conseguenza di questa maggiore attenzione storica Reinhold formula, già nel primo quaderno dei Beyträge, una teoria generale sul manifestarsi di nuove soluzioni del compito della filosofia che confortano quell'articolazione di cui si diceva: la legge generale parrebbe essere che chi rinnova profondamente il sapere filosofico, per ciò stesso non può operare, o non può farlo in maniera matura, quella movenza di secondo livello che è la fondazione del sapere. Sarebbe come dire che in filosofia ci troveremmo sempre necessariamente in una sorta di passo a due, tra iniziatore e fondatore della conoscenza. Reinhold indica come esempi di quest'innovazione che non è arrivata a tradursi in fondazione della conoscenza, Socrate nella filosofia greca e Bacone come primo esponente della rinascita della filosofia in età moderna ${ }^{14}$. Così come nel mancato sviluppo o, peggio, nel fraintendimento da parte di Aristotele e Wolff, dei vertici attinti da Platone e Leibniz, si può spiegare come l'obiettivo approssimato di soluzione ultima del compito della filosofia sia stato mancato. Possiamo pensare che, guardando a questa legge generale, Reinhold possa leggere esattamente in questo senso il compito di approfondimento e comprensione in cui è impegnato rispetto al realismo di Bardili, secondo un approccio, abbiamo detto, originale e non scevro di osservazioni critiche e ricerca di chiarimento. L'articolazione della rivoluzione filosofica, il suo essere in itinere e in via di esplicazione, non impedisce di poter assumere uno sguardo filosofico con il

13 Cf. REINHOLD, 2017, p. 38-39 per la giustificazione della trattazione da un punto di vista storico dei diversi indirizzi di pensiero post-kantiani, e p. 92-140 per lo svolgimento di questa parte sotto il titolo «l kantiani».

14 Cf. B/p. 9-10. 
quale leggere retrospettivamente l'intero corso della storia della filosofia, l'unico senso e interesse che Reinhold aveva riconosciuto alla storia della filosofia nel saggio teorico del 1796 prima ricordato. E al di là dell'idea di progresso, su questo aspetto della storia della filosofia, sensata solo se filosofica, Reinhold è del tutto in linea con i suoi critici idealisti, Schelling e Hegel ${ }^{15}$.

\section{Linee per un'interpretazione della storia della filosofia: da Platone a Leibniz al realismo logico}

Leggere la storia della filosofia in termini di progresso verso un ultimo tentativo riuscito non implica per nulla che tale progresso sia lineare. Questo emerge certamente nella valutazione di intere epoche: la filosofia ellenistica e la filosofia romana segnano la crisi del pensiero antico e per Reinhold non sono meritevoli di attenzione ${ }^{16}$; lo stesso vale per tutta l'epoca medievale da cui emerge come primo esponente del pensiero moderno Bacone, che si muove per Reinhold in un contesto di ignoranza e falso sapere ${ }^{17}$. Anche nei rapporti tra i diversi sistemi filosofici da prendere in considerazione come tentativi di soluzione del primo compito della filosofia si evidenziano vertici e crisi, che però comportano un'analisi più attenta dei singoli sistemi e dei loro concetti fondamentali.

Il criterio viene sempre dalle indicazioni teoriche sulla soluzione del compito della filosofia: un primo criterio lo abbiamo già ricordato, è quello dell'unica verità reale che tenga insieme logica, metafisica e matematica, che è però ancora un metro generale di carattere, potremmo dire, formale; un secondo criterio riguarda il merito della soluzione del compito, ed è definito dal rapporto tra due termini chiave, il «vero (Wahre)» e il «vero originario (Urwahre)». Nel saggio Was heißt

15 In Über den Begriff der Geschichte der Philosophie Reinhold scandisce la differenza tra conoscenza storica e conoscenza filosofica come differenza tra conoscenza attraverso percezione e conoscenza attraverso ragionamento: cf. REINHOLD, 1796, p. 11; egli definisce in conseguenza la storia della filosofia come «esposizione dell'insieme delle trasformazioni che la scienza della relazione necessaria delle cose ha conosciuto dal suo sorgere fino ai nostri tempi» (ivi pp. 19-20).

${ }_{16}$ Cf. $B /$ p. 6.

17 Cf. B/pp. 8-9. Per una ricostruzione più articolata del passaggio dal pensiero medievale a quello moderno cf. BV p. 128-130. 
Philosophiren? Was war es, und was soll es seyn? nel primo quaderno dei Beyträge, Reinhold indica come punto di partenza del filosofare, che è anche il punto di contatto con il senso comune, l'amore e la fede nella verità: non c'è conoscenza e quindi tensione verso la sua fondazione che non presupponga la verità come suo termine di riferimento, con il che Reinhold già esclude ogni forma di scetticismo a oltranza, negatore di ogni istanza di verità. Nello stesso tempo la fondazione come compito della filosofia implica, nel momento in cui riesce, l'abbandono del piano della fede ${ }^{18}$. Questo punto di partenza che il filosofo sviluppa nel suo lavoro di fondazione presuppone quindi anche la distinzione di verità ed errore e l'idea del vero come ciò in cui prima si crede e cui poi si attinge con il sapere al quale il filosofo tende ${ }^{19}$. Qui intervengono i due termini chiave prima ricordati: il filosofo nello scomporre l'evidenza coscienziale di un vero fuori di sé e indipendente dalla coscienza arriva ad articolare un «vero» che è vero «in sé (an sich), ma non attraverso se stesso (durch sich selbst)» e un vero che è «vero in sé e attraverso se stesso e attraverso cui tutto ciò che è vero ha la sua verità, il vero originario» ${ }^{20}$. Espresso in questi termini il vero originario potrebbe ancora essere un principio fondativo di carattere soltanto epistemologico. Che non sia così lo dice, in questo stesso contesto, il complesso degli elementi descrittivi del vero originario: il vero originario come fondamento originario o anche, secondo una ripetuta espressione reinholdiana, «Prius $\kappa \alpha \tau^{\prime} \varepsilon^{\prime} \xi o \chi \eta ́ v »$, è indipendente dal sapere tanto da essere al di fuori di esso, «inconcepibile, inspiegabile e innominabile», condizione di possibilità e realtà, quindi termine di riferimento del sapere irriducibile ad esso. Concepito in chiave ontologica, sia pure di un'ontologia che usa con prudenza concetti come «essere»o «sostanza», il vero originario attesta il vero o si manifesta in esso ed il vero, come principio del sapere equivalente all'orizzonte di conoscibilità dell'umano, è un termine

18 Cf. B/pp. 67-68.

19 Cf. $B /$ p. 70.

20 B/p. 71. 
intermedio che riconosce il vero originario sopra di sé e include sotto di sé tutto il conoscibile. ${ }^{21}$

Quest'ultimo criterio, definibile, in termini non reinholdiani, come centrato sulla differenza ontologica, unito al criterio dell'unità di logica, metafisica e matematica, cambia in maniera significativa il giudizio sul corso della filosofia. In dipendenza da Bardili, Reinhold vede in Platone e Leibniz i vertici del pensiero nella filosofia antica e nella filosofia moderna, vertici poi occultati dallo sviluppo, piuttosto da intendersi come corruzione, dato ai loro edifici di pensiero rispettivamente da Aristotele e da Wolff ${ }^{22}$.

Per Platone, dato l'oggetto dei saggi storici, non c'è una trattazione organica che ne giustifichi il primato: dal confronto con Aristotele è chiara la preferenza data alla dialettica platonica rispetto alla logica aristotelica che ha separato definitivamente nella storia della logica la forma del giudizio dal suo oggetto ${ }^{23}$. Ma, ancora, per la differenza tra vero e vero originario, Platone fornisce la struttura ontologica della differenza tra modello e copia, una struttura che fa capo all'unità come primo, alla quale Reinhold si riferisce evocando i luoghi della Repubblica di Platone nei quali Platone fa valere l'unità come base dell'intera comprensione del mondo con il conseguente apprezzamento dell'insegnamento matematico nella sua paideia ideale ${ }^{24}$.

21 «Poiché e nella misura in cui il vero originario in sé inconcepibile può manifestarsi in un vero concepibile come tale, perciò $e$ in tanto anche il filosofare non può muovere dal vero originario inconcepibile, ma da un vero concepibile. Ma questo vero concepibile, nel sapere cercato, deve condurre da un lato immediatamente al vero originario sopra di lui, dall'altro però a tutto il restante vero concepibile sotto di lui» (B I p. 73).

22 Soprattutto nei primi due quaderni dei Beyträge insistentemente Reinhold indica questa linea e il realismo di Bardili come il suo coronamento: proprio l'essere pervenuta alla rivelazione del vero originario nel vero fa della soluzione del compito della filosofia nel Grundriss la soluzione «che ha ripresentato e compiuto quella ricerca della realtà della conoscenza introdotta da Platone e proseguita ulteriormente da Leibniz» (B/p. 89). In alcuni luoghi (cf. B / p. 12, B /I p. XI-XII) Reinhold associa anche Descartes per le ragioni che vedremo subito; per ulteriori menzioni della linea Platone-Leibniz cfr. $B / p . X, 42,132 ; B$ // p. 6-7, 33; BIV p. V; B V p. 86.

23 Nei termini più chiari questa contrapposizione è scandita all'inizio del saggio Die Simplicität der Philosophie im Gegensatz mit der Duplicität der Philodoxie nel quarto quaderno dei Beyträge: alla dialettica platonica come «scienza puramente razionale ... dottrina della ragione e dell'essenza» Reinhold oppone la loro differenziazione in Aristotele con cui iniziò «la soggettivazione metodica del pensiero o della ragione nella logica e l'oggettivazione del soggettivo nella natura in metafisica» (B IV p. 213).

24 Cf. B/p. 106. 
Più articolato il discorso per Leibniz. Anche per Leibniz Reinhold parrebbe dipendere dalle valutazioni date da Bardili, anche se, diversamente da Bardili, non insiste sul tema dell'universalità e oggettività delle leggi logiche. Per mettere in luce l'originalità dell'interpretazione reinholdiana bisogna guardare a Leibniz come esito del razionalismo moderno e quindi vederlo come momento di soluzione di alcune intuizioni cartesiane rimaste però imperfette. In questo senso Descartes è nella visione della storia della filosofia di Reinhold una sorta di vertice ulteriore, e quindi gli va dedicata, in questo contesto, qualche considerazione.

Descartes, secondo una visione ampiamente condivisa anche all'epoca, è visto come un capostipite del pensiero moderno, tuttavia, e questa è già una convinzione meno diffusa, in relazione proprio con Bacone: mentre Bacone ha riportato in auge la conoscenza, Descartes, proprio grazie a questo primo passo, si è concentrato sulla fondazione della conoscenza e della conoscenza filosofica, in un orizzonte già più liberato dagli errori della cultura precedente ${ }^{25}$, un'ulteriore conferma di quella lettura articolata delle rivoluzioni filosofiche prima ricordata. Diversamente che in precedenza, Descartes è subito connesso a Platone e Leibniz e la sua soluzione del primo compito della filosofia posta al di sopra di quella della scuola leibniziano-wolffiana, questo perché il principio della conoscenza, prima di ogni sua validazione, viene riportato al vero originario, cioè posto in dipendenza del riconoscimento della divinità ${ }^{26}$. Rispetto a letture che all'epoca sottolineavano come novità fondamentale del cartesianismo il primato del cogito e quindi del soggetto come criterio di conoscenza, Reinhold invece mette in evidenza la non autosufficienza del cogito: di fronte al dubbio estremo dell'inganno, il tema del Dio ingannatore e poi del genio maligno, risulta chiaro che il cogito vale come principio «solo se e in quanto con l'esser pensato della propria esistenza, come di soggetto pensante, è connesso inscindibilmente l'esser pensato di un altro ente, dell'ente assolutamente necessario - l'esser pensato di un ente che è vero assolutamente attraverso se stesso - attraverso il quale è immediatamente vero il suo essere pensato, e attraverso il cui essere pensato

25 Cf. $B /$ p. 11.

26 Cf. $B /$ p. 13-14. 
viene garantito anche l'essere pensato dell'io pensante come tale» ${ }^{27}$. Che in questi termini la filosofia cartesiana esprima lo stesso nucleo contenutistico lo conferma la trasponibilità nei termini propri del realismo logico, del vero e del vero originario ${ }^{28}$.

Il limite che Reinhold rileva in Descartes va riportato all'epoca storica, ma in senso diverso da quello riferito a Bacone. I problemi si concentrano sul modo di pensare Dio, sia pure posto all'origine della validità del soggetto conoscente e dei suoi criteri guida nel conoscere: per Reinhold Descartes nel suo pensare Dio non ha sufficientemente elevato Dio al di sopra di tutto il restante ideale e reale, non lo ha rappresentato nella sua totale assolutezza. Reinhold critica, con queste osservazioni, l'argomento per la dimostrazione dell'esistenza di Dio che presenterebbe il problema dell'articolazione di possibilità e realtà non tenendo conto che Dio è presupposto di ogni possibilità e realtà e in questo senso non può essere assimilato agli altri oggetti della mente. Viceversa Descartes ha pensato che la differenza di Dio da ogni altro oggetto potesse essere salvaguardata dall'assunzione della realtà semplicemente a partire dalla sua possibilità ${ }^{29}$. La critica sollevata nei confronti dell'argomento ontologico cartesiano investe naturalmente anche altre e successive forme di dimostrazione dello stesso tipo e consiglia di non intendere immediatamente il nesso logica-metafisica di Reinhold come un ritorno al razionalismo prekantiano. E Reinhold stesso, sempre in questo contesto, appare consapevole del carattere originale della sua critica. Essa si ripercuote poi sul concetto di sostanza: Descartes si sarebbe illuso attraverso l'eccedenza dell'idea di Dio evidenziata dall'argomento ontologico, di poter usare il concetto di sostanza tanto per Dio quanto per il finito, ed è su questo terreno che Spinoza, pur segnando un regresso nella posizione del primo compito della filosofia, lo mette in mora, in quanto mostra la contraddittorietà dell'idea cartesiana di sostanza e la sua esclusiva riferibilità, a rigore, a Dio e non al finito ${ }^{30}$. La concezione di Dio come unica sostanza non è risolutiva, mantiene la natura oggettuale di Dio rispetto

27 B/p. 15.

28 Cf. $B /$ p. 12-13.

29 Cf. $B /$ p. $20-21$.

30 Cf. B / p. 23-24. Per una riproposizione di questi stessi argomenti cf. B V p. 131-133.

Rev. Filos., Aurora, Curitiba, v. 30, n. 51, p. 727-750, set./dez. 2018 
alla quale in questo contesto Reinhold indica nel realismo logico la via d'uscita rispetto all'alternativa oggettività-soggettività incarnata rispettivamente da Spinoza e da Fichte ${ }^{31}$.

Si capisce allora, sulla base degli elementi sottolineati da Reinhold, in che senso Leibniz è un momento conclusivo di questo processo: la filosofia leibniziana riporta la conoscenza a Dio come suo principio nello stesso senso valorizzato in Descartes ${ }^{32}$. Anche se Reinhold introducendo la trattazione di Leibniz avverte che il concetto di sostanza non può essere considerato il concetto centrale della filosofia leibniziana e sarebbe affrettato quindi collocare Leibniz del tutto dentro il confronto tra Descartes e Spinoza, rimane il fatto che una parte considerevole della sua sintesi e dei riferimenti testuali a Leibniz riguardano questo concetto. Reinhold ne mette in evidenza tratti significativi quali l'essere la sostanza forza rappresentante, il che consente di estendere il concetto all'intero mondo senza escluderne Dio e anche di porre in relazione la sostanza divina alle sostanze finite; la scansione della differenza tra sostanze in termini di qualità, di rappresentazioni con diverso grado di consapevolezza che determinano le differenze tra animale e uomo; la crucialità della sostanza divina come base dell'intero mondo. Così quando tira un bilancio delle acquisizioni della filosofia leibniziana, è la concezione della sostanza in termini di forza rappresentante ciò che si aggiunge in modo decisivo al già noto rapporto di vero e vero originario ${ }^{33}$. Questo nucleo è venuto meno, secondo l'interpretazione reinholdiana, perché Wolff ne ha lasciato cadere i motivi essenziali, cogliendone la lettera e non lo spirito, irrigidendola in un sistema che riproduce una vecchia scolastica e condizionandone così la recezione ed anche l'oblio.

31 Cf. B/p. 23.

32 Cf. $B / p .27,35,41-42$.

33 «È incontestabile che lo spirito della filosofia leibniziana risiede nello sforzo di fondare la realtà della conoscenza nell'unico modo possibile, vale a dire mediante la presentazione del vero originario nel vero e insieme mediante la derivazione del vero dal vero originario ... Inoltre la particolare caratteristica della filosofia leibniziana consiste nel fatto che essa spiega l'essenzialità dell'oggetto reale, o della sostanza, in generale l'essenza di ogni monade, come una forza che rappresenta l'universo» (B I p. 39). 


\section{II confronto critico con la filosofia contemporanea: criticismo e idealismo}

È il rapporto con la filosofia contemporanea, dal criticismo all'idealismo fino ai suoi esiti ultimi in Schelling, quello più profondamente modificato dalla svolta realistica e dai nuovi criteri di valutazione della storia della filosofia. Dopo essere stato parte di questa vicenda filosofica, Reinhold ne prende radicalmente distanza, facendo anche di se stesso oggetto di storia come qualcosa di ormai trascorso ${ }^{34}$.

Se Kant era stato il punto terminale delle controversie filosofiche dell'intera storia del pensiero, ora è al più uno snodo della rinascita del pensiero in Germania rispetto all'eclettismo della Popularphilosophie, Reinhold è anche disposto a riconoscergli un'approssimazione alla linea Platone-Leibniz che, con un diverso contesto, avrebbe potuto permettergli di compiere l'attesa rivoluzione filosofica e di questo trova chiare tracce nella concezione kantiana della ragione nella dialettica trascendentale delle prime due Critiche ${ }^{35}$, ma per altro verso Kant non ha saputo attingere un principio unificante del suo sistema filosofico e soprattutto si è chiuso in quel circolo centrato sul soggetto per il quale Reinhold conia il fortunato termine di «soggettività», come principio che accomuna criticismo e idealismo.

Per ciò che riguarda l'acquisizione di un unico principio per la fondazione della conoscenza della verità, Reinhold lamenta la separazione che interviene nella logica trascendentale kantiana tra elemento soggettivo-formale e materia del conoscere, in ultimo la cosa in sé $\mathrm{e}^{36}$. E quanto all'impianto complessivo della filosofia critica, se nei precedenti apprezzamenti della filosofia kantiana Reinhold aveva fatto valere il passaggio dal piano teoretico a quello pratico come chiave di soluzione delle controversie metafisiche, qui nei Beyträge rinnega la praticabilità

34 Reinhold dedica un paragrafo della seconda parte del saggio Die erste Aufgabe der Philosophie, in ihren merkwürdigsten Auflösungen, seit der Wiederherstellung der Wissenschaften nel secondo quaderno dei Beyträge alla propria Elementarphilosophie, quindi al proprio lavoro di fondazione del criticismo, collocandosi in quella parte del kantismo che aveva inteso andare oltre il criticismo come propedeutica alla filosofia prima, trattando poi nello stesso paragrafo le critiche scettiche di Gottlob Ernst Schulze e Salomon Maimon (cf. B II p. 35-43).

35 Cf. B /l p. 6-7. Per le valutazioni su Kant come snodo di una rinascita della filosofia in Germania cf. B I p. XI, 4.

36 Cf. B/p. 17. 
di una metafisica su base morale: né il carattere assoluto della legge né la distinzione tra arbitrio e libertà possono celare la soggettività come radice ultima dell'agire, e il postulato di Dio su base morale non va oltre la posizione di un «come se» Dio fosse, che equivale in ultimo alla negazione stessa di Dio ${ }^{37}$. Tanto meno è riconosciuta alla Kritik der Urteilskraft una capacità di sintesi rispetto ai due ambiti teoretico e pratico $^{38}$, al punto che essa non è ritenuta meritevole di una trattazione autonoma accanto alle altre due Critiche.

È chiaro che rispetto al criterio che ha guidato la rilettura del razionalismo moderno, il rapporto del vero con un vero originario inteso ontologicamente, cioè fuori dall'uomo e dal suo sapere, tanto da essere inconcepibile, inspiegabile e innominabile, la filosofia di Kant, al di fuori di quei cenni alla ragione prima ricordati, rimane chiusa nel cerchio del soggetto ed è riportabile al principio della soggettività di cui lo stesso Kant non è venuto in chiaro. In ambito conoscitivo Kant attinge ad una realtà della conoscenza soltanto relativa e non riconducibile a nulla di assoluto ${ }^{39}$, e Reinhold nel secondo quaderno dei Beyträge riassume il senso ultimo della Kritik der reinen Vernunft nel fatto che «la verità in generale sarebbe solo soggettiva, e che la verità conosciuta in modo puro, la verità filosofica, consisterebbe nella soggettività conosciuta in modo puro, come pura verità» ${ }^{40}$. In ambito pratico la ragione kantiana abbandona la dipendenza dall'oggetto che l'aveva caratterizzata in campo conoscitivo, non abbandona invece il carattere della soggettività, anzi «il carattere dell'assolutezza che assume come pratica è semplicemente quello della soggettività assoluta o dell'assolutezza soggettiva» ${ }^{41}$, formula che Reinhold ripete quasi identica a proposito del

37 Reinhold sviluppa questa critica alla morale kantiana più ancora che nella sezione dedicata alla Kritik der praktischen Vernunft del capitolo su Kant della sua rassegna storico-filosofica, nel saggio n. III del secondo quaderno dei Beyträge, Ueber die Autonomie als Princip der praktischen Philosophie der Kantischen — und der gesammten Philosophie der Fichtischschellingschen Schule, cf. B I/ p. 104-140.

38 Cf. $B / /$ p. 11-12.

39 Cf. B/p. 79.

40 B /lp. 20.

${ }^{41}$ B/p. 80. 
carattere falsamente incondizionato della legge morale ${ }^{42}$. E la soggettività è quanto si nasconde per Reinhold dietro le condizioni materiali dell'esperienza in ambito conoscitivo e dietro l'assolutezza e necessità della legge morale ${ }^{43}$.

Reinhold non ha così difficoltà ad ammettere che Fichte, rispetto a tutti gli interpreti di Kant incluso Reinhold stesso, è stato quello che più ne ha riconosciuto il principio ispiratore, la soggettività, e lo ha portato radicalmente a compimento:

Incontestabilmente Fichte è penetrato più profondamente di Kant nel vero spirito dell'idealismo trascendentale, in quanto nella sua Wissenschaftslehre ha riconosciuto ed applicato quell'attività oggettivante se stessa della soggettività assoluta come il principio non solo della filosofia pratica, ma di ogni filosofia (idealistica) ${ }^{44}$.

Reinhold usa in molteplici luoghi i termini «soggettività», «egoità», «pura ragione», con significato equivalente, per indicare il principio della filosofia contemporanea dal criticismo all'idealismo ${ }^{45}$. Il movimento che caratterizza questo principio è quello dell' «agire che ritorna in sé (in sich zurückgehende Tun)», una «autoattività (Selbstthätigkeit)» frutto dell'arbitrio del soggetto che si concentra su se stesso, quindi riflette su di sé, previa astrazione dal mondo di oggetti fuori di lui, e così guadagna il piano dell'io filosofico, supponendo di lasciarsi alle spalle anche la sua individualità particolare. Reinhold si riferisce, anche esplicitamente, per questa descrizione alle introduzioni e all'esposizione fichtiane della Wissenschaftslehre apparse sul Philosophisches Journal tra 1797 e 1798, in particolare a quei luoghi nei quali Fichte invita all'esercizio del pensarsi per guadagnare il primato dell'intuizione intellettuale e la connessione di intuizione intellettuale e intuizione sensibile ${ }^{46}$. E trova, nello stesso saggio n. III del secondo

42 «Il datore di questa legge è solo la ragione stessa, come assoluta autoattività — come assolutezza della soggettività e soggettività dell'assolutezza» (B I p. 138).

43 Cf. $B / /$ p. 26.

$44 \quad$ B/p. 82.

45 Cf. $B /$ p. 141 per la loro chiara equiparazione.

46 Cf., per l'utilizzzzione soprattutto della Zweyte Einleitung in die Wissenschaftslehre, B // p. 45 e sgg. e cf. anche ivi p. 107-108. 
quaderno dei Beyträge, conforto nei saggi schellinghiani dei volumi 6 e 7 sempre del Philosophisches Journal nei quali Schelling riconosce a Fichte questo stesso merito dell'individuazione di un primo principio della filosofia kantiana e lo sviluppa a sua volta ragionanando sull'assolutezza della libertà ${ }^{47}$. In questo senso Fichte e Schelling hanno in comune e hanno sviluppato lo stesso principio della soggettività ${ }^{48}$. Nel saggio del primo quaderno dei Beyträge nel quale descrive e critica questo principio, Ideen zu einer Heautogonie oder natürlichen Geschichte der reinen Ichheit, genannt, reine, Vernunft, Reinhold tratta in totale parallelo i due filosofi idealisti e rivolge loro lo stesso rimprovero che rimarrà costante anche nelle trattazioni successive: ciò che nel movimento di costituzione della soggettività è rimasto celato ai loro occhi è il meccanismo psicologico che guida l'intero movimento, il desiderio di indipendenza assoluta e il rifiuto di dipendere da altri connaturato all'essere umano. La conseguenza è che al fondo di tutto questo c'è proprio l'individuo empirico dal quale Fichte e Schelling pretendevano di sollevarsi e pretendevano di sollevare anche i loro interlocutori se dotati di capacità filosofiche ${ }^{49}$.

Al tempo stesso, anche per essersene precocemente occupato, Reinhold aveva ben presente che Schelling aveva percorso una via originale all'idealismo elaborando una filosofia della natura, e già nel primo quaderno dei Beyträge dà una giustificazione teorica di questo: Schelling si sarebbe reso conto dell'insostenibilità di una visione dell'assoluto come soggettività e avrebbe spinto così il principio della soggettività fino all'estremo creativo di un movimento astrattivo che produce la natura. Reinhold mette così in parallelo, facendo tesoro dell'interpretazione dell'idealismo data in ultimo da Schelling nel System des transzendentalen Idealismus, dottrina del sapere e dottrina della natura come «scienze fondamentali di un'unica e medesima filosofia ${ }^{50}$, teorizzando una piena convertibilità di idealismo e materialismo che mostra neanche troppo velatamente di avere dietro il nesso spinozismo-idealismo

\footnotetext{
47 Cf. $B / /$ p. 105 et seq.

48 «È toccato a Fichte e Schelling trasformare l'intera filosofia in un atto libero dell'uomo» (B II p. 122).

49 Cf. B/p. 153-154, B II p. 112-113.

$50 \quad$ B/p. 87 e cf. ivi p. 85-88.
} 
del Sendschreiben an Fichte di Jacobi, che aveva formulato l'idea dell'idealismo fichtiano come spinozismo rovesciato. Nel secondo quaderno dei Beyträge questa differenza assume più precisi connotati filologici e storici: il giovane Schelling già nell'elaborazione della sua prima filosofia si sarebbe giovato dei progressi realizzati da Fichte e ciò lo avrebbe portato a concepire la sua fisica speculativa, merito che Reinhold afferma come il maggiore e più proprio di Schelling. La filosofia della natura schellinghiana fa fronte in modo più convincente alle incongruenze della trattazione fichtiana della natura, che per Reinhold è costretta ad abbassarsi al punto di vista della coscienza comune: ne sarebbero esempi il modo di Fichte di trattare il mondo naturale in Über den Grund unseres Glaubens an eine göttliche Weltregierung (1798) o il ricorso alla nozione di fede nella Bestimmung des Menschen (1800), critica quest'ultima condivisa da Schelling e Hegel in questi stessi anni ${ }^{51}$.

I motivi di critica a Schelling rimangono comunque tutti e ritornano anche là dove Reinhold si trova a fare i conti con l'ultimo stadio della filosofia schellinghiana, la filosofia dell'identità proposta anzitutto nella Darstellung meines Systems del 1801. Reinhold prende atto dell'abbandono dell'idea di due vie parallele all'idealismo sostenuta da Schelling fino al 1800 e del tentativo di Schelling di ricavare soggettivo e oggettivo da un unico principio, leggendolo come un segno di quella stessa inconseguenza che aveva afflitto Fichte. È anche chiaro a Reinhold che questa nuova prospettiva rende la filosofia schellinghiana più contigua al realismo logico e quindi concorrente. Segno di questo è il confronto con la filosofia schellinghiana in un saggio pubblicato in due parti nel terzo e quarto quaderno dei Beyträge $e^{52}$. Al di là della chiara insinuazione di una dipendenza della filosofia dell'identità

51 Cf. $B$ /l p. 63-64.

52 Cf. Ueber das absolute Identitätssystem, oder der neuesten reinen Rationalismus des Herrn Schelling und dessen Verhältnis zum rationalen Realismus, B III p. 163-184, e Schlüssel zur Philodoxie überhaupt und insbesondere zur sogenannten Spekulativen, B IV p. 186-201, indicato da Reinhold come prosecuzione del precedente, cui segue (cf. B IV p. 202-211) una risposta all'aspro articolo contro la filosofia di Reinhold pubblicato da Schelling sul primo volume del Kritisches Journal, Über das absolute Identitäts-System, und sein Verhältnis zum neuesten (Reinholdischen) Dualismus. Ein Gespräch zwischen dem Verfasser und einem Freund. 
dal realismo logico di Bardili ${ }^{53}$, nel merito filosofico per Reinhold tutto si gioca sulla definizione della ragione, il punto di partenza nella costruzione del sistema della Darstellung meines Systems. La differenza, argomenta Reinhold, sta nel fatto che l'identità del realismo logico, il pensiero come pensiero, non coincide con l'essere originario, non viene qualificata, come succede in Schelling e in modo immotivato, come assoluta, e in questo carattere di identità non ulteriormente specificata manifesta e dà a conoscere Dio e l'essenza delle cose, salvaguarda la rivelazione di Dio che per il Reinhold dei Beyträge è rivelazione nella natura più ancora che nella coscienza morale nell'agire dell'uomo. In Schelling viceversa identità ed essere si sovrappongono e l'identità è totalità, smarrendo completamente il riferimento fondamentale della differenza tra vero e vero originario ${ }^{54}$. Ancora di più quindi la filosofia di Schelling è interpretabile come compimento dell'idealismo in termini di «filodossia», piena sostituzione di una parvenza al vero originario. Anche i soli termini di questo confronto polemico però fanno intravedere un campo di discussione più ampio e complesso: se l'esito dell'idealismo è così contiguo con il realismo logico allora è proprio nella definizione, entro i confini teorici del realismo logico, dell'identità sul piano logico nel suo nesso con il reale (nel linguaggio del realismo logico, l'applicazione del pensiero alla materia) che si gioca il confronto tra idealismo e realismo. L'ampio disegno reinholdiano entro i Beyträge della definizione del rapporto tra analisi come primo compito della filosofia, dottrina elementare del realismo, e fenomenologia come analisi della natura, versante applicato e compimento di quella dottrina elementare, costituiscono il necessario complemento del confronto qui descritto nel suo versante più polemico e storico-filosofico ${ }^{55}$.

53 Per il passaggio dall'idea di un parallelismo di idealismo e materialismo, quindi un parallelismo di Fichte e Spinoza, alla loro identità, Reinhold insinua che si potrebbe pensare che per quei due occhi portati a identità attraverso un paio di occhiali «il signor Schelling si sia fatto fare quegli occhiali da Bardili) (B III p. 168) se non ci fossero le sue rassicurazioni di aver elaborato quest'idea in un tempo precedente, quindi prima della pubblicazione del Grundriss.

54 Cf. $B$ III p. 169-171.

55 Reinhold stesso lega programmaticamente nella premessa al terzo quaderno Beyträge la sua nuova esposizione degli elementi del realismo razionale (il saggio $\mathrm{n}$. III dello stesso quaderno, cf. B III p. 128-162) alla fenomenologia del realismo razionale del quaderno successivo (cf. B IV p. 104-185). II combinato di sistema compiuto della filosofia pura e fenomenologia come nesso del realismo con i fenomeni è destinato a lasciarsi dietro la speculazione dell'identità del 
C'è un ultimo aspetto del confronto con la filosofia contemporanea, rimasto fin qui implicito e che merita menzionare in conclusione. Per tutto quanto detto la logica-metafisica perseguita da Reinhold ridefinisce il limite della filosofia, affermando la non esauribilità sul piano della conoscenza del vero originario nella sua differenza e relazione col vero. Ne viene di conseguenza la necessità di chiarire il posto della fede nella filosofia, nozione che abbiamo visto intervenire metodicamente nella posizione del compito della filosofia ed anche, in relazione a Fichte, nel giudizio sulla filosofia contemporanea. Questo tema inquadra il rapporto del realismo logico con la filosofia del tutto peculiare di Friedrich Heinrich Jacobi. Nel paragrafo dedicato a Jacobi nella seconda parte, nel secondo quaderno dei Beyträge, del saggio Die erste Aufgabe der Philosophie, in ihren merkwürdigsten Auflösungen, Reinhold indica senza equivoci in Jacobi la figura che nell'epoca contemporanea più si approssima alla linea Platone-Leibniz ${ }^{56}$, ne richiama la precoce e corretta comprensione dei limiti della filosofia kantiana e la speculare rivalutazione del razionalismo prekantiano e la decisa opposizione ad ogni pretesa dimostrativa del vero originario. Nel giudizio di Reinhold, con riferimento all'ultima produzione jacobiana, in particolare al Sendschreiben an Fichte, Jacobi avrebbe mostrato che «quell'Altro e più alto dovrebbe essere ciò che è elevato (das Erhabene) per antonomasia oltre ogni soggettività e oggettività, il vero originario in sé inconcepibile ed inesprimibile, che non potrebbe essere né la nostra ragione né la natura in generale, che si manifesta però in entrambe come donatore della ragione e origine della natura - e attraverso il cui riconoscimento soltanto la ragione in noi viene costituita come vera ragione» ${ }^{57}$. Il centro del realismo logico nell'interpretazione reinholdiana è chiaramente riconoscibile, ma è anche chiaro che questa diversa idea di ragione prospetta un accesso

soggettivo e dell'oggettivo come parvenza (cf. B III p. IV-V). E a conclusione di un breve scritto di giustificazione sulle proprie conversioni filosofiche, Rechenschaft über mein Systemwechseln, Reinhold indica nell'opposizione tra filosofia dell'identità schellinghiana e realismo suo e di Bardili I'ultima alternativa con cui ha che fare la filosofia nella comprensione del pensiero (cf. $B V$ p. 23-46, in particolare p. 45-46).

56 Cf. $B$ // p. 33, e Reinhold richiama l'epiteto per Jacobi di «Platone tedesco» per l'originalità e bellezza dell'esposizione letteraria (cf. $B / /$ p. 28).

57 $B / /$ p. 32-33. 
immediato, un'immediata rivelazione del vero originario antagonista alla via che Reinhold propone. E va ricordato che proprio a ridosso dell'adesione al realismo logico, nel Sendschreiben an J. K. Lavater und J. G. Fichte über den Glauben an Gott, lo scritto con il quale in parallelo al Sendschreiben an Fichte di Jacobi, Reinhold era intervenuto nell'Atheismusstreit relativo alla filosofia di Fichte, Reinhold nella fase forse di massima prossimità a Jacobi aveva ricercato un punto di vista intermedio tra il non sapere di Jacobi e il sapere di Fichte ${ }^{58}$. Reinhold ha ormai abbandonato l'idea di questo punto di vista intermedio e semmai giudica la difesa jacobiana del punto di vista della fede come effetto di una sua mancata comprensione della rivoluzione introdotta dal Grundriss der ersten Logik di Bardili ${ }^{59}$. Egli ribadisce questa sua posizione nei Briefe an F. H. Jacobi. Ueber das Wesen der Jacobischen, Fichteschen, Schellingschen und Bardilischen Philosophie, una serie di lettere a Jacobi pubblicate sul quinto quaderno dei Beyträge ${ }^{60}$. Come comunica lo stesso Reinhold in avvio della prima lettera, del 9 settembre 1802, l'occasione di questa serie di lettere è la discussione critica della filosofia di Jacobi nel Kritisches Journal, precisamente nel saggio hegeliano Glauben und Wissen, e la critica ivi condotta della fede jacobiana come figura estrema della soggettività finita ${ }^{61}$. La difesa del punto di vista jacobiano della fede rispetto agli esiti dell'idealismo, ma anche la risoluzione della fede jacobiana nel più alto punto di vista del realismo logico, dice quanto questo snodo sia decisivo per Reinhold, al punto che per dedicarvisi, come afferma sempre in avvio della prima lettera, si è liberato dei suoi incarichi istituzionali come prorettore all'università di Kiel ${ }^{62}$.

Anche su questo versante per Reinhold si gioca non soltanto il punto della filosofia a inizio del XIX secolo, restituito in questi imponenti affreschi di cui abbiamo dato conto, ma anche il suo immediato avvenire.

58 Cf. REINHOLD, 1799, p. 6-7.

59 Cf. $B / /$ p. 33.

${ }^{60}$ Cf. $B$ V p. 69-114.

${ }^{61}$ Cf. $B \vee$ p. 69.

62 Cf. ibidem. 


\section{Riferimenti bibliografici}

BARDILI, C. G. Grundriss der ersten Logik gereiniget von den Irrthümmern bisheriger Logiken überhaupt, der kantischen insbesondere; keine Kritik sondern eine Medicina mentis, brauchbar hauptsächlich für Deutschlands Kritische Philosophie. Stuttgart: Löflund, 1800.

HEGEL, G. W. F. Jenaer kritische Schriften. Hg. H. Buchner und O. Pöggeler. Hamburg: Meiner, 1968. (Gesammelte Werke, Band 4).

KANT, I. Kritik der reinen Vernunft. Hamburg: Meiner, 1956.

REINHOLD, K. L. Über den Begriff der Geschichte der Philosophie. In: FÜLLEBORN, G. G. (Hrsg.). Beiträge zur Geschichte der Philosophie. Züllichau u. Freystadt: Frommann, 1796. p. 3-36. I u. II Stück.

REINHOLD, K. L. Sendschreiben an J. K. Lavater und J. G. Fichte über den Glauben an Gott. Hamburg: Perthes, 1799.

REINHOLD, K. L. Beyträge zur leichtern Uebersicht des Zustandes der Philosophie beym Anfange des 19. Jahrhunderts. Hamburg: Perthes, 1801-1803. v. 1-6.

REINHOLD, K. L. Auswahl vermischter Schriften. Zweiter Theil. Hrsg. M. Bondeli und S. Imhof. Basel: Schwabe, 2017. (Gesammelte Schriften. Kommentierte Ausgabe, Band 5/2).

Ricevuto: 23/08/2018

Received: 08/23/2018

Recebido: 23/08/2018

Approvato: 20/09/2018 Approved: 09/20/2018 Aprovado: 20/09/2018 